\title{
Angular momentum transport and large eddy simulations in magnetorotational turbulence: the small Pm limit
}

\author{
Heloise Meheut ${ }^{1}$, Sébastien Fromang ${ }^{1}$, Geoffroy Lesur ${ }^{2,3}$, Marc Joos ${ }^{1}$, and Pierre-Yves Longaretti ${ }^{2,3}$ \\ ${ }^{1}$ Laboratoire AIM, CEA/DSM-CNRS-Université Paris 7, Irfu/Service d'Astrophysique, CEA-Saclay, 91191 Gif-sur-Yvette, France \\ e-mail: heloise.meheut@cea.fr \\ 2 Univ. Grenoble Alpes, IPAG, 38000 Grenoble, France \\ 3 CNRS, IPAG, 38000 Grenoble, France
}

Received 19 January 2015 / Accepted 19 May 2015

\begin{abstract}
Context. Angular momentum transport in accretion discs is often believed to be due to magnetohydrodynamic turbulence mediated by the magnetorotational instability (MRI). Despite an abundant literature on the MRI, the parameters governing the saturation amplitude of the turbulence are poorly understood and the existence of an asymptotic behaviour in the Ohmic diffusion regime has not been clearly established.

Aims. We investigate the properties of the turbulent state in the small magnetic Prandtl number limit. Since this is extremely computationally expensive, we also study the relevance and range of applicability of the most common subgrid scale models for this problem. Methods. Unstratified shearing box simulations are performed both in the compressible and incompressible limits, with a resolution up to 800 cells per disc scale height. This is the highest resolution ever attained for a simulation of MRI turbulence. Different magnetic field geometry and a wide range of dimensionless dissipative coefficients are considered. We also systematically investigate the relevance of using large eddy simulations (LES) in place of direct numerical simulations.

Results. In the presence of a mean magnetic field threading the domain, angular momentum transport converges to a finite value in the small Pm limit. When the mean vertical field amplitude is such that $\beta$ (the ratio between the thermal and magnetic pressure) equals $10^{3}$, we find $\alpha \sim 3.2 \times 10^{-2}$ when Pm approaches zero. In the case of a mean toroidal field for which $\beta=100$, we find $\alpha \sim 1.8 \times 10^{-2}$ in the same limit. Implicit LES and the Chollet-Lesieur closure model both reproduce these results for the $\alpha$ parameter and the power spectra. A reduction in computational cost by a factor of at least 16 (and up to 256) is achieved when using such methods.

Conclusions. MRI turbulence operates efficiently in the small Pm limit provided there is a mean magnetic field. Implicit LES offers a practical and efficient means of investigation of this regime but should be used with care, particularly in the case of a vertical field. The Chollet-Lesieur closure model is perfectly suited for simulations done with a spectral code.
\end{abstract}

Key words. accretion, accretion disks - magnetohydrodynamics (MHD) - turbulence - protoplanetary disks - methods: numerical

\section{Introduction}

Determining the rate of angular momentum transport in accretion discs is considered to be one of the most important unsolved astrophysical questions. Accretion is believed to be at the origin of the radiation emitted by some of the most luminous sources in the universe from active galactic nuclei to X-ray binaries and is also a major process at work during planet formation in protoplanetary discs (Frank et al. 2002). In addition, accretion discs are ubiquitous in the universe and affect the dynamics, evolution, and appearance of multiple astrophysical objects at all spatial and energy scales. The accretion rate can be indirectly constrained by the luminosity of high-energy sources or the lifetime of protoplanetary discs, and, if the transport process is modelled by an $\alpha$ viscosity (Shakura \& Sunyaev 1973), such an estimate gives $0.01<\alpha<0.4$ depending on the system considered (King et al. 2007).

The physical origin of angular momentum transport has to be understood to explain such an efficient radial angular momentum transport. Currently, the most widely accepted mechanism is magnetohydrodynamic (MHD) turbulence induced by the non-linear evolution of the magneto-rotational instability (MRI, Balbus \& Hawley 1991). That instability along with its non-linear development has been extensively studied in the last two decades (Balbus \& Hawley 1998; Balbus 2003; Fromang 2013). Using local simulations performed in the framework of the shearing box, Hawley et al. (1995) quickly established that the MRI develops into vigorous MHD turbulence that efficiently transports angular momentum outward, a result that was later confirmed to be independent of the field geometry (Hawley et al. 1996) or to the background disc stratification (Brandenburg et al. 1995; Stone et al. 1996). Only recently has the sensitivity of MRI-driven MHD turbulence saturation to small-scale dissipation in such idealized simulations been identified: when magnetic field diffusion is dominated by an ohmic resistivity $\eta, \alpha$ is an increasing function of the magnetic Prandtl number $P m$, the ratio between the kinematic viscosity $v$ and $\eta$. This is the case both in the presence of a mean vertical magnetic field (Lesur \& Longaretti 2007) and of a mean azimuthal magnetic field (Simon \& Hawley 2009). Such a "Pm-effect" has a significant impact on the rate of angular momentum transport measured in homogeneous shearing box simulations: in the case of a mean vertical field such that the plasma $\beta$ parameter (defined in Sect. 2.4) amounts to $10^{3}$, Longaretti \& Lesur (2010) showed that $\alpha$ varies by a factor of about 5 when $P m$ only varies between $1 / 4$ and 4, without any sign of the relation flattening at either range. The dynamo case (i.e. no mean magnetic field threading the computational domain) also displays a high sensitivity to $\mathrm{Pm}$ 
(Fromang et al. 2007; Simon \& Hawley 2009; Guan et al. 2009; Bodo et al. 2011) that persists when density stratification is included (Simon et al. 2011). In this dynamo regime and without stratification, the effect is even stronger and dynamo action is suppressed for $P m$ values smaller than unity, in which case the flow remains laminar, i.e. $\alpha$ goes to zero. A clear understanding of the origin of the effect of $\mathrm{Pm}$ on the rate of angular momentum transport is still lacking and is currently a matter of active research. The first results, based on methods borrowed from the fluid community (Herault et al. 2011; Riols et al. 2013) are promising (Riols et al. 2015) and should be extended to more realistic geometries and dimensionless numbers.

Taken together, the results described above question the relevance of MRI-driven MHD turbulence as the dominant transport mechanism in accretion discs where the Prandtl number can be orders of magnitude lower $(P m \ll 1)$ than has been explored in published simulations (Balbus \& Henri 2008). There is clearly the possibility that $\alpha$ becomes vanishingly small as $\mathrm{Pm}$ decreases to small but astrophysically relevant values. The first goal of this paper is to investigate this asymptotic behaviour by means of high-resolution simulations performed in the homogeneous shearing box. In doing so, we will leave aside the dynamo case and focus on vertical and azimuthal mean field configurations. Using high-resolution simulations (such that the coverage in Pm now extends over more than two orders of magnitudes, from $10^{-2}$ to 4 ), we will show that $\alpha$ asymptotically converges to a well-defined finite value in both cases. However, the computational cost associated with such simulations is extremely high (for example, 15 million CPU hours are needed to complete the $1000^{3}$ simulation we describe in Sect. 3.1.1 on a BlueGen/Q machine ranked 42nd on the Top500 supercomputing website in November $2014^{1}$ ). In practice, such a high cost is prohibitive if one wants to perform a parameter survey in the asymptotic regime with additional physics included and simply prevents global simulations from being performed in that limit. The second goal of this paper is thus to investigate the possibility of using sub-grid scale models as a means of reducing the computational cost associated with MHD turbulence simulations in the small Pm limit.

Recent years have seen significant progress in our understanding of the consequences of ambipolar diffusion (Bai \& Stone 2011, 2013; Simon et al. 2013) and the Hall effect (Kunz \& Lesur 2013; Lesur et al. 2014; Bai 2015), both of which are particularly important in shaping the structure of protoplanetary discs. In this paper we will restrict our attention to Ohmic resistivity as the sole source of magnetic field dissipation. Such a regime is relevant in order to describe the very inner parts of protoplanetary discs (at stellar distances of a few tens of an AU) and also cataclysmic variable $(\mathrm{CV})$ discs and the outer parts of $\mathrm{X}$-ray binary discs (Balbus \& Henri 2008). In principle, the analysis we present here should also be carried for such cases where ambipolar diffusion or the Hall effect is the dominant magnetic field diffusion processes.

The plan of the paper is as follows. In the following section, the equations and numerical methods are given. Section 3 presents our results, focusing first on simulations that explicitly resolve the small dissipative scales (Sect. 3.1) and then on two different methods for performing large eddy simulations (LES) in Sect. 3.2. We finally conclude and discuss the implications of our work in Sect. 4.

\footnotetext{
See http://www. top500.org/list/2014/11/
}

\section{Methods}

\subsection{Equations and notations}

In this paper we use the shearing box approximation (Hawley et al. 1995). Namely, we compute the evolution of the fluid in a small box centred at a radius $r_{0}$ of the disc and rotating at the same velocity as the fluid at $r=r_{0}$. As the size of the box is small compared to the radial position, the curvature terms of the standard fluid equations can be simplified (Hawley \& Balbus 1992). We thus use Cartesian coordinates $(x, y, z)$ with unit vectors $(\boldsymbol{i}, \boldsymbol{j}, \boldsymbol{k})$. In this coordinate system, we denote $\left(L_{x}, L_{y}, L_{z}\right)$ the size of the computational box. Neglecting the vertical component of the gravitational acceleration, we solve the following set of equations,

$\partial_{t} \rho+\nabla \cdot(\rho \boldsymbol{v})=0$

$\partial_{t}(\rho \boldsymbol{v})+\boldsymbol{\nabla} \cdot(\rho \boldsymbol{v} \boldsymbol{v}-\boldsymbol{B} \boldsymbol{B})+\boldsymbol{\nabla} P_{\text {tot }}=2 q \rho \Omega_{0}^{2} x \boldsymbol{i}-2 \rho \boldsymbol{\Omega}_{0} \times \boldsymbol{v}+\boldsymbol{\nabla} \cdot \boldsymbol{T}$

$\partial_{t} \boldsymbol{B}=\boldsymbol{\nabla} \times(\boldsymbol{v} \times \boldsymbol{B}-\eta \boldsymbol{\nabla} \times \boldsymbol{B})$

where $\rho$ is the fluid density, $v$ its velocity in the rotating frame, $\boldsymbol{B}$ is the magnetic field, and $\Omega_{0}$ is the angular velocity of the fluid at $r_{0}$ corresponding to the angular velocity of the box; $q$ stands for the background Keplerian shear and is taken as equal to 1.5 throughout this paper; $P_{\text {tot }}$ is the total pressure, the sum of the thermal pressure $P$ and the magnetic pressure $B^{2} / 2$. Explicit dissipation is accounted for through the Ohmic resistivity $\eta$ and the kinematic viscosity $v$ that enters in the viscous stress tensor $\boldsymbol{T}$ defined as

$T_{i j}=\rho v\left(\partial_{j} v_{i}+\partial_{i} v_{j}-\frac{2}{3} \delta_{i j} \boldsymbol{\nabla} \cdot \boldsymbol{v}\right)$.

The amplitude of viscosity and resistivity are set using the magnitude of the Reynolds number $R e$ and magnetic Reynolds number $R m$ with the relations

$R e=\frac{\Omega_{0} L_{z}^{2}}{v}, R m=\frac{\Omega_{0} L_{z}^{2}}{\eta}$,

which can also serve as an alternative definition for the magnetic Prandtl number $P m$ already given in the introduction:

$P m=\frac{v}{\eta}=\frac{R m}{R e}$.

We performed simulations that consider two different flavours of the system of Eqs. (1)-(3). First, we solved the above equations in the incompressible limit. In this case, the density is constant and Eq. (1) reduces to $\boldsymbol{\nabla} \cdot \boldsymbol{v}=0$. We used the code SNOOPY in that case (see Sect. 2.2). In the second type of simulations, we solved the full set of equations (and refer to that case as the compressible simulations) using the code RAMSES (Sect. 2.3). We briefly describe below the two codes and the specificities of each set of simulations.

\subsection{Incompressible simulations: the SNOOPY code}

The SNOOPY code is a pseudo-spectral code that solves the incompressible MHD equations in a Fourier basis. It uses a low-storage third-order Runge-Kutta integrator and works in a sheared frame comoving with the mean flow, which is equivalent to a third-order Fargo scheme (Masset 2000). SNOOPY uses a $3 / 2$ antialiasing rule to eliminate the excitation of spurious modes during the computation of quadratic non-linearities.

The use of a sheared Fourier basis makes the boundary conditions periodic in the $y$ - and $z$-directions and shear-periodic in 
the $x$-direction. SNOOPY conserves linear momentum and magnetic flux down to machine precision. Since spectral methods are inherently diffusion free and energy conserving, the addition of dissipation is required to mimic the damping due to small scale dissipation processes ${ }^{2}$. In SNOOPY, one can choose secondorder diffusion operators, hyperdiffusion operators, or Chollet \& Lesieur (1981) subgrid models (see Sect. 3.2.1).

\subsection{Compressible simulations: the RAMSES code}

The RAMSES code is a finite volume code that solves the compressible MHD equations on a Cartesian grid (Teyssier 2002; Fromang et al. 2006) using the constrained transport algorithm (Evans \& Hawley 1988). We use a version of the code for which the grid is uniform (i.e. without the adaptive mesh refinement). The source terms associated with the tidal potential are included as described by Stone \& Gardiner (2010). We use the HLLD Riemann solver (Miyoshi \& Kusano 2005) with monotonized central slope limiter, shearing box boundary conditions in the $x$-direction (Hawley et al. 1995), and periodic boundary conditions in the $y$ - and $z$-directions. For extended box sizes such as used in the mean vertical field case (see Sect. 2.4), it has been found that radially variable numerical dissipation causes the turbulent stress to vary accross the box. To avoid that problem, we used the FARGO algorithm (Masset 2000; Stone \& Gardiner 2010 ) in that case, which also improves the efficiency of the code through an increase in the timestep.

Throughout this paper, we use an isothermal equation of state to close the system of MHD equations in compressible simulations, such that $P=\rho c_{0}^{2}$, where $c_{0}$ stands for the constant sound speed. We start the simulation with an initial uniform density $\rho=\rho_{0}$ and choose $L_{z}=H=c_{0} / \Omega_{0}$, where $H$ is the disc scale height.

\subsection{Models parameters}

We start the simulations with a uniform initial magnetic field:

$\boldsymbol{B}=B_{0 y} \boldsymbol{j}+B_{0 z} \boldsymbol{k}$.

Two different initial magnetic configurations are considered in the following, namely pure azimuthal field $\left(B_{0 z}=0\right)$ simulations and pure vertical field simulations $\left(B_{0 y}=0\right)$. The strength of the magnetic field is defined using the plasma parameter $\beta_{i}$ that is given by

$\beta_{i} \equiv \begin{cases}\frac{q^{2} \rho_{0} c_{0}^{2}}{B_{0 i}^{2}} & \text { in compressible runs, } \\ \frac{\left(q \Omega L_{z}\right)^{2}}{B_{0 i}^{2}} & \text { in incompressible runs. }\end{cases}$

For the mean azimuthal (resp. vertical) magnetic field simulations, we used $\beta_{y}=112.5$ (resp. $\beta_{z}=10^{3}$ ). At the start of each run, small amplitude random velocity perturbations are added on the three velocity components with an amplitude equal to $1 \%$ of the sound speed in compressible runs. In incompressible simulations both velocity and magnetic random perturbations are added with an amplitude of $0.1 \Omega L_{z}$.

\footnotetext{
2 The numerical stability of the scheme does not require any dissipation. However, the absence of dissipation in a turbulent flow naturally leads to thermalization, a situation which does not occur in natural systems, which always exhibit dissipation processes. Numerical dissipation is therefore required on physical grounds to break the thermodynamic equilibrium and create the well-known energy cascade picture.
}

The size of the computational box is either $\left(L_{z}, 4 L_{z}, L_{z}\right)$ for the simulations with a mean azimuthal magnetic field, or $\left(4 L_{z}, 4 L_{z}, L_{z}\right)$ for the simulations with a mean vertical magnetic field. In the latter case, it is indeed well known that boxes with $L_{x}=L_{z}$ artificially enhance the importance of recurrent bursts in the flow structure (Bodo et al. 2008; Johansen et al. 2009). In such box sizes and with such vertical magnetic field, Bai \& Stone (2014) recently reported zonal flows. We also found such structures in our simulations.

The resolution varies from 32 cells per unit length up to 800 cells per unit length. Such high resolutions allow us to reach the largest Reynolds number (run Y-C-Re85000, $R e=$ 85000 ) and the smallest Prandtl number (run Z-I-Re40000, $P m=0.01)$ ever published. As larger structures are expected in the $y$-direction, we typically use a resolution that is twice as coarse in that direction.

\section{Results}

The whole set of runs discussed in the remainder of this paper is listed in Tables 1-3, where the first column provides the simulation labels. Runs starting with a pure azimuthal (vertical) magnetic field are labelled with the letter "Y" ("Z"). Likewise, compressible (incompressible) simulations are labelled with the letter "C" ("I"). Large eddy simulations are labelled either "ILES" or "CL" depending on the subgrid scale model that is used (see Sect. 3.2). The remaining columns in Tables $1-3$ give the run resolution (Col. 2) and duration $T_{\text {Run }}(\mathrm{Col} .3)$, the Reynolds number (Col. 4), the magnetic Reynolds number (Col. 5), the magnetic Prandtl number (Col. 6), and $\alpha$ (Col. 7), which is the sum of $\alpha_{\text {Rey }}$ (Col. 8) and $\alpha_{\text {Max }}$ (Col. 9). The last two are defined by the following relations:

$$
\alpha_{\text {Rey }}= \begin{cases}\frac{\left\langle\rho \delta v_{r} \delta u_{\phi}\right\rangle}{P_{0}} & \text { in compressible runs, } \\ \frac{\left\langle\delta u_{r} \delta u_{\phi}\right\rangle}{(\Omega L z)^{2}} & \text { in incompressible runs, }\end{cases}
$$

$\alpha_{\text {Max }}= \begin{cases}-\frac{\left\langle B_{r} B_{\phi}\right\rangle}{P_{0}} & \text { in compressible runs, } \\ -\frac{\left\langle B_{r} B_{\phi}\right\rangle}{\rho_{0}(\Omega L z)^{2}} & \text { in incompressible runs. }\end{cases}$

Here $\langle$.$\rangle denotes an average over the shearing box volume and$ over time and $\delta v$ is the velocity difference to the laminar sheared flow. Except for the shortest runs (see Sect. 3.1.1), the turbulent transport rates as measured by the $\alpha$ parameters are time averaged over the last 60 orbits of the models. The last column gives the ratio between magnetic and hydrodynamic transport rate.

\subsection{Direct numerical simulations in the small $\mathrm{Pm}$ regime}

In this section, we present the results of the resolved simulations (meaning that kinematic viscosity and ohmic resistivity are explicitly included in the calculation), focusing on the rate of angular momentum transport and on the power spectra of the turbulent flow.

\subsubsection{A $1000^{3} \mathrm{MRI}$ simulation}

Because of the tremendous computational cost that was associated with that simulation, we start with a description of model Y-C-Re85000, performed with RAMSES. In this model, $R e=$ 85000 and $P m=0.03$. This simulation was performed with a resolution $\left(N_{x}, N_{y}, N_{z}\right)=(800,1600,800)$. Based on past experience and published results of simulations using the same 
Table 1. RAMSES runs with a mean azimuthal field.

\begin{tabular}{cccccccccc}
\hline \hline Model & Resolution & $T_{\text {Run }}$ & $R e$ & $R m$ & $P m$ & $\alpha$ & $\alpha_{\text {Max }}$ & $\alpha_{\text {Rey }}$ & $R$ \\
\hline Y-C-Re650 & $(64,128,64)$ & 100 & 650 & 2600 & 4 & $3.0 \times 10^{-2}$ & $2.4 \times 10^{-2}$ & $5.9 \times 10^{-3}$ & 4.2 \\
Y-C-Re2600 & $(64,128,64)$ & 100 & 2600 & 2600 & 1 & $2.5 \times 10^{-2}$ & $1.9 \times 10^{-2}$ & $5.7 \times 10^{-3}$ & 3.4 \\
Y-C-Re13000 & $(128,256,128)$ & 100 & 13000 & 2600 & 0.2 & $1.8 \times 10^{-2}$ & $1.4 \times 10^{-2}$ & $4.6 \times 10^{-3}$ & 3.0 \\
Y-C-Re26000 & $(256,512,256)$ & 100 & 26000 & 2600 & 0.1 & $2.0 \times 10^{-2}$ & $1.5 \times 10^{-2}$ & $5.0 \times 10^{-3}$ & 3.0 \\
Y-C-Re85000 & $(800,1600,832)$ & 35 & 85000 & 2600 & 0.03 & $1.8 \times 10^{-2}$ & $1.3 \times 10^{-2}$ & $4.6 \times 10^{-3}$ & 2.9 \\
\hline Y-ILES-C-64 & $(64,128,64)$ & 100 & - & 2600 & - & $1.9 \times 10^{-2}$ & $1.4 \times 10^{-2}$ & $4.7 \times 10^{-2}$ & 3.0 \\
Y-ILES-C-128 & $(128,256,128)$ & 100 & - & 2600 & - & $1.9 \times 10^{-2}$ & $1.4 \times 10^{-2}$ & $4.8 \times 10^{-3}$ & 3.0 \\
Y-ILES-C-256 & $(256,512,256)$ & 100 & - & 2600 & - & $1.9 \times 10^{-2}$ & $1.4 \times 10^{-2}$ & $5.0 \times 10^{-3}$ & 2.8 \\
\hline
\end{tabular}

Notes. The box size is $\left(L_{x}, L_{y}, L_{z}\right)=\left(L_{z}, 4 L_{z}, L_{z}\right) . T_{\text {run }}$ is given in units of local orbits. For each run the table gives its resolution, duration, Reynolds number, magnetic Reynolds number, magnetic Prandtl number, total stress, Maxwell stress, Reynolds stress, and the ratio of the two stresses.

Table 2. Same as Table 1, but for RAMSES runs with a mean vertical field.

\begin{tabular}{cccccccccc}
\hline \hline Model & Resolution & $T_{\text {Run }}$ & $R e$ & $R m$ & $P m$ & $\alpha$ & $\alpha_{\text {Max }}$ & $\alpha_{\text {Rey }}$ & $R$ \\
\hline Z-C-Re400 & $(128,64,32)$ & 100 & 400 & 400 & 1 & $6.5 \times 10^{-2}$ & $4.0 \times 10^{-2}$ & $2.4 \times 10^{-2}$ & 1.6 \\
Z-C-Re800 & $(256,128,64)$ & 100 & 800 & 400 & 0.5 & $5.1 \times 10^{-2}$ & $2.9 \times 10^{-2}$ & $2.2 \times 10^{-2}$ & 1.3 \\
Z-C-Re3000 & $(512,256,128)$ & 100 & 3000 & 400 & 0.13 & $3.7 \times 10^{-2}$ & $2.0 \times 10^{-2}$ & $1.8 \times 10^{-2}$ & 1.1 \\
Z-C-Re8000 & $(1024,512,256)$ & 100 & 8000 & 400 & 0.05 & $3.1 \times 10^{-2}$ & $1.5 \times 10^{-2}$ & $1.6 \times 10^{-2}$ & 1.0 \\
\hline Z-ILES-C-32 & $(128,64,32)$ & 100 & - & 400 & - & $4.1 \times 10^{-2}$ & $2.2 \times 10^{-2}$ & $1.9 \times 10^{-2}$ & 1.2 \\
Z-ILES-C-64 & $(256,128,64)$ & 100 & - & 400 & - & $3.6 \times 10^{-2}$ & $1.8 \times 10^{-2}$ & $1.8 \times 10^{-2}$ & 1.0 \\
Z-ILES-C-128 & $(512,256,128)$ & 100 & - & 400 & - & $3.3 \times 10^{-2}$ & $1.6 \times 10^{-2}$ & $1.7 \times 10^{-2}$ & 1.0 \\
Z-ILES-C-256 $^{*}$ & $(1024,512,256)$ & 40 & - & 400 & - & $3.3 \times 10^{-2}$ & $1.6 \times 10^{-2}$ & $1.8 \times 10^{-2}$ & 0.9 \\
\hline
\end{tabular}

Notes. The box size is $\left(L_{x}, L_{y}, L_{z}\right)=\left(4 L_{z}, 4 L_{z}, L_{z}\right){ }^{(*)}$ To save computational time, this model was performed by restarting model Z-C-Re8000 at $t=50$ removing explicit viscosity.

Table 3. Same as Table 1, but for SNOOPY runs.

\begin{tabular}{cccccccccc}
\hline \hline Model & Resolution & $T_{\text {Run }}$ & $R e$ & $R m$ & $P m$ & $\alpha$ & $\alpha_{\text {Max }}$ & $\alpha_{\text {Rey }}$ & $R$ \\
\hline Z-I-Re1300 & $(256,256,64)$ & 53 & 1333 & 400 & 0.3 & $3.8 \times 10^{-2}$ & $2.8 \times 10^{-2}$ & $9.9 \times 10^{-3}$ & 2.8 \\
Z-I-Re20000 & $(1536,768,192)$ & 53 & 20000 & 400 & 0.02 & $3.5 \times 10^{-2}$ & $2.6 \times 10^{-2}$ & $9.2 \times 10^{-3}$ & 2.8 \\
Z-I-Re40000 & $(3072,1536,384)$ & 53 & 40000 & 400 & 0.01 & $3.3 \times 10^{-2}$ & $2.3 \times 10^{-2}$ & $1.0 \times 10^{-2}$ & 2.4 \\
\hline Z-CL-192-a & $(768,384,192)$ & 53 & - & 400 & - & $3.3 \times 10^{-2}$ & $2.4 \times 10^{-2}$ & $8.8 \times 10^{-3}$ & 2.8 \\
Z-CL-192-b & $(768,384,192)$ & 53 & - & 400 & - & $3.2 \times 10^{-2}$ & $2.3 \times 10^{-2}$ & $9.3 \times 10^{-3}$ & 2.5 \\
Z-CL-128-a & $(512,256,128)$ & 53 & - & 400 & - & $3.5 \times 10^{-2}$ & $2.4 \times 10^{-2}$ & $1.0 \times 10^{-2}$ & 2.4 \\
Z-CL-128-b & $(512,256,128)$ & 53 & - & 400 & - & $3.4 \times 10^{-2}$ & $2.5 \times 10^{-2}$ & $9.7 \times 10^{-3}$ & 2.5 \\
Z-CL-96-a & $(384,192,96)$ & 53 & - & 400 & - & $3.4 \times 10^{-2}$ & $2.5 \times 10^{-2}$ & $9.2 \times 10^{-3}$ & 2.8 \\
Z-CL-96-b & $(384,192,96)$ & 53 & - & 400 & - & $3.7 \times 10^{-2}$ & $2.6 \times 10^{-2}$ & $1.1 \times 10^{-2}$ & 2.3 \\
\hline
\end{tabular}

Notes. All runs have a box size of $\left(L_{x}, L_{y}, L_{z}\right)=\left(4 L_{z}, 4 L_{z}, L_{z}\right)$.

set-up at a lower Reynolds number with a similar code (Simon $\&$ Hawley 2009), we are confident that the small scales of the flow are properly accounted for in this simulation. In the $x$ - and $z$-directions, the number of cells thus amounts to 800 cells per disc scale height, which is the highest resolution ever achieved of MRI-driven MHD turbulence with a second-order compressible code. This gigantic simulation was run at the IDRIS supercomputing centre on the BlueGen-Q machine Turing, using 32768 cores. We found that the most efficient configuration was to use 4 threads per core, meaning that a total of 131072 threads (or, equivalently, MPI sub-domains) were used. We used approximately $10 \mathrm{~h}$ of CPU time per timestep. Running the simulation for over 35 orbits $\left(\sim 10^{6}\right.$ timesteps) thus required about $10^{7} \mathrm{~h}$ of CPU time, corresponding to $300 \mathrm{~h}$ of wall clock time (i.e. about two weeks).
As described by Simon \& Hawley (2009), in the presence of a mean azimuthal magnetic field, resistivity can prevent the linear instability from transiting to a turbulent state when the simulation starts from a laminar flow, even though it is found to remain turbulent on long timescales when that linear phase is bypassed. We thus adopted the method described by these authors and performed the run in two steps. We first solved the ideal MHD equations (i.e. with vanishing viscosity and resistivity parameters). The only dissipative effects are numerical in origin during that part of the calculation. A turbulent state is reached after $\sim 10$ orbits. The turbulent transport as measured by $\alpha$ displays fluctuations around a well-defined mean value of about $6 \times 10^{-2}$ for the next few orbits (see Fig. 1, top panel). At $t=15$, we restarted the simulation for an additional 20 orbits with dissipation coefficients such that $R e=85000$ and $P m=0.03$. As seen 

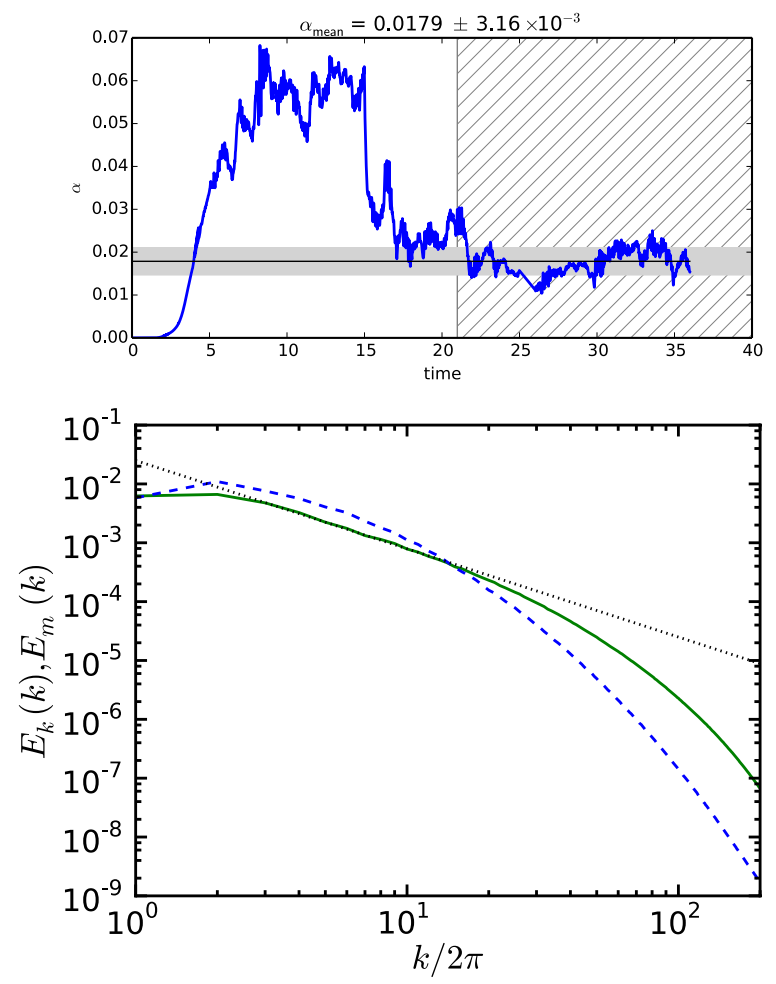

Fig. 1. Top panel: time evolution of $\alpha$ in model Y-C-Re85000 for which $P m=0.03$. The initial 15 orbits were performed in the ideal MHD regime, before including explicit diffusion coefficients. The hatched region represents the time interval over which the angular momentum transport is averaged. Bottom panel: Y-C-Re85000 run, kinetic (solid green line) and magnetic energy (dashed blue line) power spectrum.

in the top panel of Fig. 1, the turbulence amplitude is quickly modified by the presence of those dissipation coefficients and reaches a new steady state after a few additional orbits ${ }^{3}$. The nature of the flow is illustrated by a series of snapshots of the last time step of the run in Fig. 2. On the density plot one can recognize large-scale density waves and low-amplitude shocks. The turbulent magnetic field is dominated by large-scale structures. On the contrary, the velocity snapshot shows both largeand small-scale structures, as expected given the very small Pm used here.

We next averaged the transport rate after the system had reached a quasi-steady state and until the end of the simulation. This period corresponds to $t>21$ orbits and is hatched in Fig. 1 (top panel). The values of $\alpha_{\text {Rey }}, \alpha_{\text {Max }}$, and $\alpha$ that we obtained are $4.6 \times 10^{-3}, 1.3 \times 10^{-2}$, and $1.8 \times 10^{-2}$, respectively (see also Table 1). This $\alpha$ value is comparable to the one reported by Simon \& Hawley (2009) for their most resolved run, for which $P m=0.25, R m=3200$, and $\beta=450$. The Maxwell and Reynolds stresses display a ratio of $\sim 3$ that is typical of such simulations. There is of course a degree of arbitrariness regarding the exact time at which we decide that the system has reached the "quasi-steady state" and start averaging. We have checked that the statistics we consider do not vary significantly when making modest changes to the averaging period. For example, we find $\alpha=1.9 \times 10^{-2}$ and $\alpha=1.8 \times 10^{-2}$ when averaging over the periods $t>18$ and $t>25$ orbits, respectively. This is only a

\footnotetext{
3 All the runs with a mean azimuthal magnetic field are executed using the same procedure. However, except for model Y-C-Re85000 for which the computational cost is considerable, they are usually run for 100 orbits to obtain a more precise measure of $\alpha$.
}

variation of $7 \%$ and it gives an idea of the uncertainty associated with that measurement.

To obtain a more accurate view of the energy budget at each scale, we also show in Fig. 1 (bottom panel) the time averaged power spectra. Because of the shearing box boundary conditions, we consider time dependent unsheared wave vector $\boldsymbol{k}$ and the shell filter decomposition of the physical variables (Hawley et al. 1995). Because the decomposition is spherically symmetric, this method filters out the information about the flow anisotropy, which is known to be significant for MRI-driven turbulence (see Murphy \& Pessah 2015; Fig. 3 in Lesur \& Longaretti 2011, and Sect. 3.2.1). For each wavenumber, the kinetic and magnetic energy are then given by

$$
\begin{aligned}
& E_{K}(k)=\overline{v_{k}^{2}(k) / 2} \\
& E_{M}(k)=\overline{\boldsymbol{B}_{k}^{2}(k) / 2},
\end{aligned}
$$

respectively, where the bar denotes an average over time. Magnetic energy dominates at large scales $\left(k^{\prime}<15\right.$, where we have defined $k^{\prime}=k / 2 \pi$ so that it corresponds to scales $l^{\prime}>H / 15$ ), while kinetic energy dominates at smaller scales. This is a signature of the small $P m$ value of the simulation, which implies that the resistive dissipation length is larger than the viscous dissipation length (Schekochihin et al. 2004). At small scales, the motions essentially correspond to hydrodynamic turbulence associated with a forward cascade (Lesur \& Longaretti 2011). We will exploit this scale separation in Sect. 3.2 when designing subgrid scale models for the hydrodynamic part of the flow. The kinetic energy power spectrum follows a power law with exponent $-3 / 2$ over the range $2<k^{\prime}<20$, thus covering one order in magnitude in spatial scales. The same exponent has been reported recently in other high-resolution simulations of MRI-driven MHD turbulence both in the dynamo regime (Fromang 2010) and in the presence of a net vertical field (Lesur \& Longaretti 2011), suggesting it is a general feature of the flow. We note that homogeneous forced MHD turbulence also displays the same exponent (Mason et al. 2008), although the result is still debated (Beresnyak 2014). The surprising result here is that the magnetic energy does not show any obvious signature of a power-law regime, contrary to the case of homogeneous and driven MHD turbulence, while still being comparable in magnitude with $E_{K}$. More work is needed to clarify the reason for this discrepancy and to better understand the origin of the $-3 / 2$ exponent that is obtained in the case of MRI-driven MHD turbulence.

\subsubsection{Angular momentum transport}

We plot in Fig. 3 the total averaged stress $\alpha$ for all the resolved simulations we performed. Error bars $\sigma_{\alpha}$ are estimated with the method presented in Longaretti \& Lesur (2010). In general, we find $\sigma_{\alpha} \sim 5 \times 10^{-3}$ for the vertical field model (thus giving $\sigma_{\alpha} / \alpha \sim 5 \%$ ). This is consistent with the estimate of Longaretti \& Lesur (2010). The error estimate is smaller in the azimuthal field case, for which we obtained $\sigma_{\alpha} \sim 10^{-3}$, which corresponds to $\sigma_{\alpha} / \alpha \sim 1 \%$. We note that we could not apply the same method for model Y-C-Re85000 because of the short duration of the integration in that case. A simple standard deviation is thus plotted instead and explains the larger error bar for that particular model.

In agreement with previous results (see Sect. 1) we find that $\alpha$ increases with Pm. However, the main result of Fig. 3 (and the main result of the paper) is that there is now convincing evidence of the convergence of the angular momentum transport rate at small Pm toward a well-defined, non-zero value. This is the case for both field geometries. 

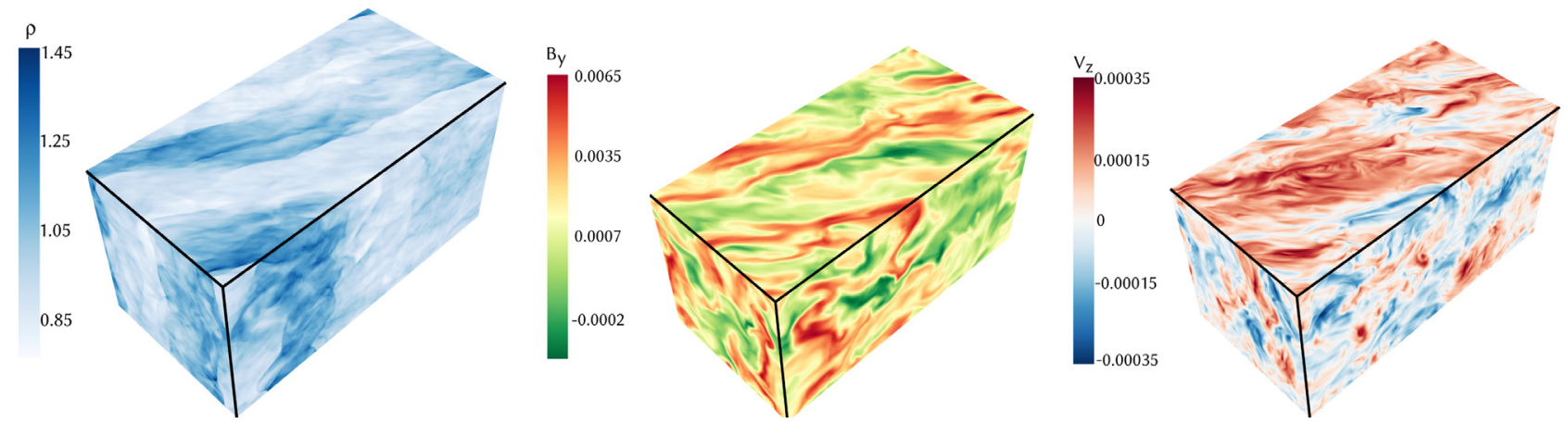

Fig. 2. From left to right, 3D snapshots of $\rho, B_{y}$, and $v_{z}$ in model Y-C-Re85000 at the end of the simulation.

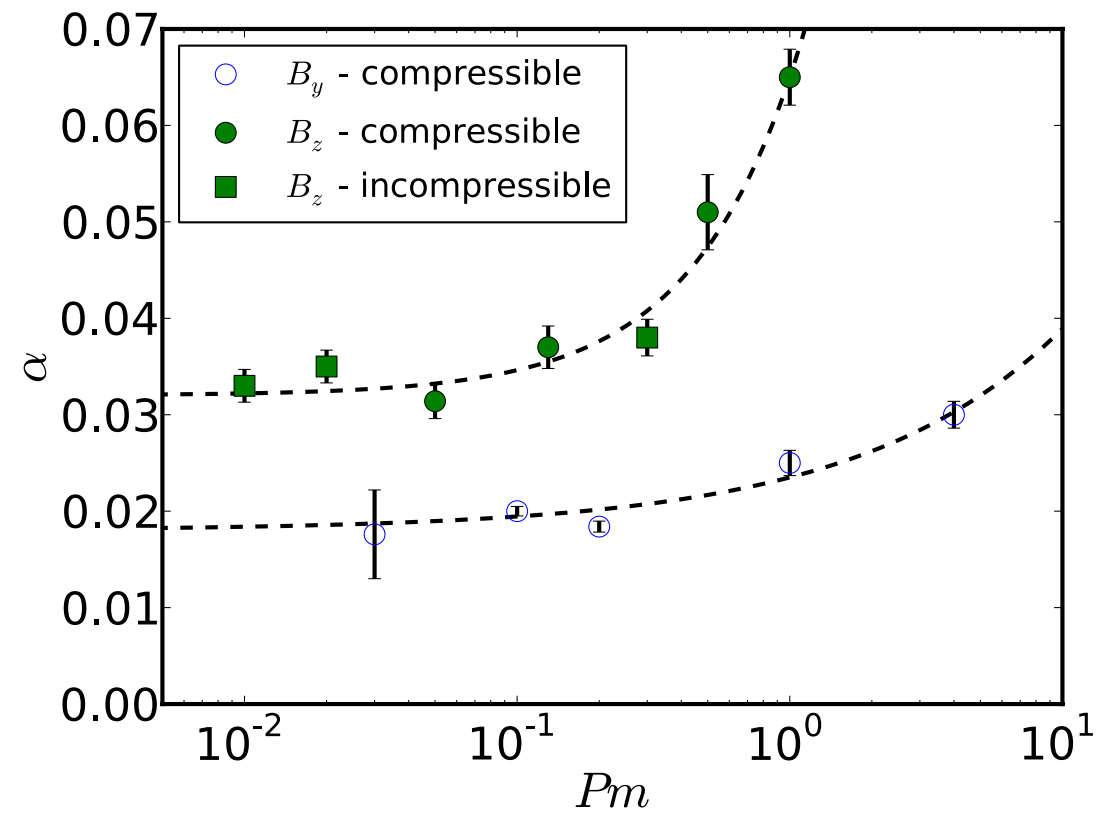

Fig. 3. Mean value of the angular momentum transport (measured by means of $\alpha$ ) for the mean toroidal field simulations (blue circles), the mean vertical field simulations performed with RAMSES (green filled circles), and with SNOOPY (green filled squares). The dashed lines are power-law functions that approximately describe the data (see text).

The azimuthal field case: in this case, and with constant $R m$ and $\beta$, we find that a fit to the data is given by the formula

$\alpha_{B y}=\alpha_{B y \min }+C_{y} P m^{0.58}$,

with the values ${ }^{4}$ of $\alpha_{B y \min }=1.8 \times 10^{-2}$ and $C_{y}=5.5 \times 10^{-3}$. This means that a good estimate of $\alpha$ is already obtained at $P m=0.2$ for which a resolution of 128 cells per unit length is sufficient. Indeed, we obtained $\alpha=1.8 \times 10^{-2}$ which is equal to the asymptotic value of $\alpha$ at vanishingly low $\mathrm{Pm}$.

The vertical field case: here, we find a somewhat larger transport coefficient that can be fitted by the relation

$\alpha_{B z}=\alpha_{B z \min }+C_{z} P m^{1.1}$

with $\alpha_{B z \min }=3.2 \times 10^{-2}$ and $C_{z}=3.3 \times 10^{-2}$, with fixed $R m$ and $\beta$. Moreover, there is good agreement between the compressible and incompressible approach in this vertical field case: for both flow types the simulations converge at small Pm toward the same $\alpha$. As is true for the azimuthal field case, a good estimate

\footnotetext{
4 The asymptotic transport values $\alpha_{B y \min }$ and $\alpha_{B z \min }$ depend in principle on $R m$ and $\beta$. See the discussion in Sect. 4.
}

of the asymptotic rate of angular momentum transport is already obtained for $P m=0.13$ (using a resolution of 128 cells per unit length), for which we found $\alpha=3.7 \times 10^{-2}$, i.e. a value that differs by about $15 \%$ from the asymptotic limit.

As already noted (Sect. 2.4), in the presence of a vertical magnetic field we find that the time history of $\alpha$ displays significant variability. This is illustrated in Fig. 4 (left panel) for the particular case of model Z-C-Re3000 ( $P m=0.13)$ : while the time averaged transport rate amounts to $\alpha=3.7 \times 10^{-2}$ in that case, there are numerous peaks during which it reaches values as high as 0.1 that occur with a typical period of about 5 to 10 orbits. Such bursts are not unheard of in unstratified shearing boxes with a mean vertical field (Bodo et al. 2008; Latter et al. 2009) and have been attributed to recurrent "channel-like" modes associated with the MRI. For the set of parameters we have considered here, the time history of $\alpha$ suggests that they contribute significantly to the turbulent transport. In an attempt to quantify that contribution, for model Z-C-Re3000 we have calculated the value $\alpha_{\text {axi }}$ of the transport that is due to axisymmetric channel-like modes (for which $k_{y}^{\prime}=0$ and $k_{z}^{\prime}=1$ ) for 120 dumps evenly spaced between $t=40$ and $t=100$. The mean value of $\alpha_{\text {axi }}$, averaged over all the dumps of the simulation, amounts to $1.2 \times 10^{-2}$; this means that axisymmetric 

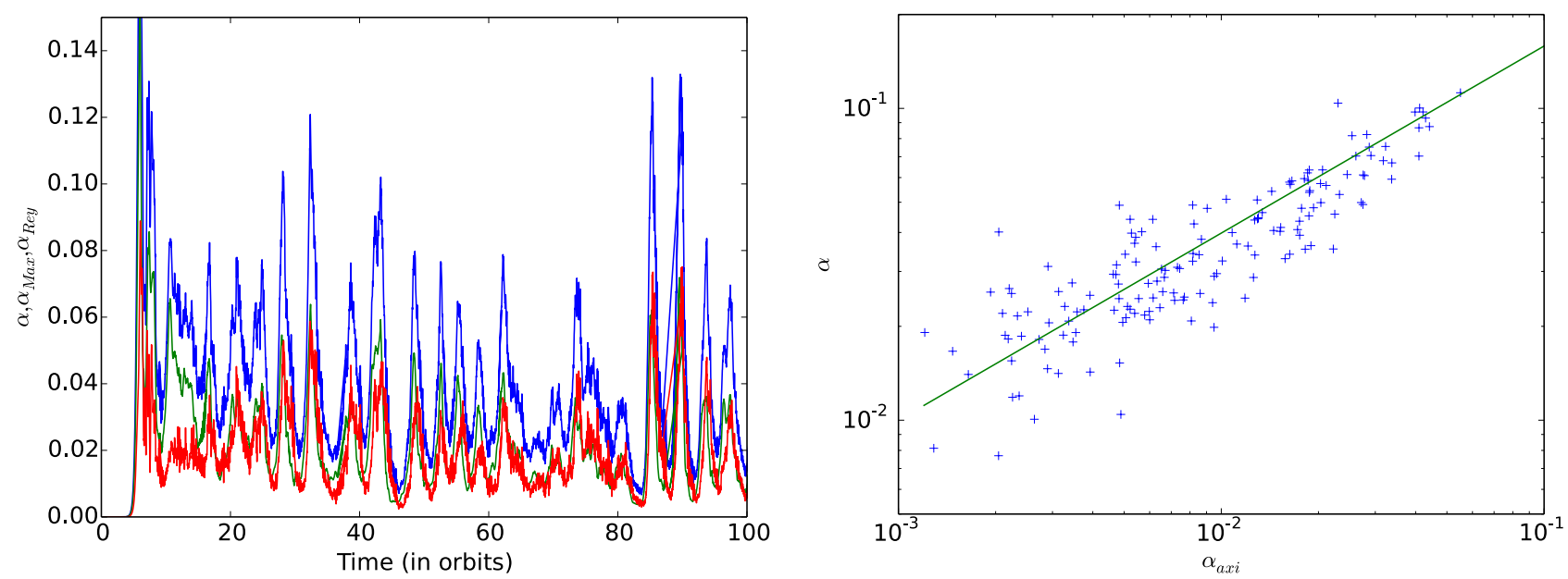

Fig. 4. Left panel: time history of $\alpha$ (blue curve), $\alpha_{\text {Max }}$ (red curve), and $\alpha_{\text {Rey }}$ (green curve) in model Z-C-Re3000. Right panel: scatter plot showing the total angular momentum transport rate $\alpha$ as a function of $\alpha_{\text {axi }}$ which measures the angular momentum transport due to $k_{y}^{\prime}=0$ and $k_{z}^{\prime}=1$ modes for 120 dumps evenly spaced over model Z-C-Re3000. The green curve plots an approximate fit to the data (see text for details).

modes with $k_{z}^{\prime}=1$ account for $\sim 30 \%$ of the angular momentum transport. In agreement with the results of Longaretti \& Lesur (2010), angular momentum transport is dominated on average by non-axisymmetric modes even if the contribution of channellike modes is significant. The scatter plot showing the relation between $\alpha$ and $\alpha_{\text {axi }}$ for those 120 dumps is shown in the right panel of Fig. 4, along with an indicative fit of the data given by

$\alpha=\alpha_{0}\left(\frac{\alpha_{\text {axi }}}{\alpha_{\text {axi0 }}}\right)^{0.6}$,

with $\alpha_{0}=10^{-2}$ and $\alpha_{\text {axio }}=10^{-3}$. The positive correlation between $\alpha$ and $\alpha_{\text {axi }}$ indicates that the relative contribution of the transport mediated by axisymmetric modes with $k_{z}^{\prime}=1$ increases during the bursts of activity. For example, $\alpha_{\text {axi }}$ amounts to only about $10 \%$ of the transport when $\alpha=10^{-2}$ but, as indicated by Eq. (15), can contribute up to $50 \%$ of the turbulent activity when $\alpha=0.1$. These variations are consistent with the results of Latter et al. (2009) and with the idea that the bursts seen in Fig. 4 are due to large-scale channel-like modes (Bodo et al. 2008). We have repeated the same analysis for all the models and we have found a weak dependance of the relative fraction of axisymmetric transport with $P m: \alpha_{\text {axi }} / \alpha=0.39,0.34,0.32$, and 0.29, respectively for $P m=1,0.5,0.13$, and 0.05 and similar results for the incompressible runs with $\alpha_{\text {axi }} / \alpha=0.38,0.35$, respectively for $P m=0.02$ and 0.01 .

It is also noteworthy that the angular momentum transport in the presence of a vertical field is equally due to Maxwell and Reynolds stresses in the compressible simulation, whereas the classical ratio of approximately 3 is obtained in an incompressible fluid or with an azimuthal field configuration for the specific values of $\beta$ and $R m$ chosen in our investigation. The exact value of the Maxwell-to-Reynolds stress ratio $(R)$ are given in the last column of Tables $1-3$. In an attempt to have a better understanding of the relative contribution of Maxwell and Reynolds stresses, we plot in Fig. 5 their time history over two burst cycles for the particular case of model Z-C-Re3000. This plot reveals that the Reynolds stress is larger than the Maxwell stress during the decaying part of the burst when the channel-like modes (with $k_{y}=0$ and $k_{z}=1$ ) are destroyed by the non-linear turbulent dynamics. The computation of the contribution of the axisymmetric modes shows that the Maxwell transport is dominated by channel-like modes whereas the Reynolds transport is due

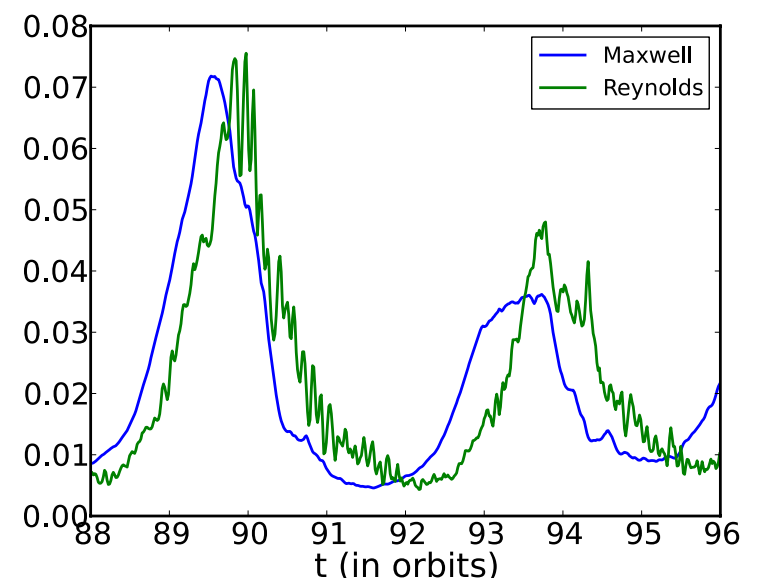

Fig. 5. Time history of $\alpha_{\text {Max }}$ (blue curve) and $\alpha_{\text {Rey }}$ (green curve) in model Z-C-Re3000.

to non-axisymmetric modes. Because of the difference between the incompressible and compressible runs, we further speculate that such a destruction is associated with the excitation of compressible modes such as density waves. However, this detailed study is not directly related to the angular momentum transport rate and is beyond the scope of this paper.

\subsection{Large eddy simulations in the small Pm limit}

The previous results have shown that angular momentum transport converges toward a well-defined limit at small Pm. However, such simulations are very computationally expensive. Here, we investigate the possibility of using a subgrid scale model instead of standard kinematic viscosity. The aim is to reduce the computational cost of the simulations without compromising the physics one may want to consider, such as the accretion rate or the turbulent power spectra.

Historically, the study of small Pm flows has mostly relied on simulations using hyperviscosity such as geodynamo models (Glatzmaiers \& Roberts 1995) or small-scale dynamo theory (Schekochihin et al. 2007). However, hyperviscosity is known to produce numerous artefacts in hydrodynamic turbulence, such as spectral bottlenecks, reduced intermittency, and spurious isotropization (Frisch et al. 2008). For this reason, 
we instead focus on LES in which the dissipation adapts dynamically to the turbulent cascade initiated by the large scales, thereby limiting the artefacts commonly due to hyperviscosity.

Large eddy simulations are routinely used in industrial applications to model flows at high $R e$. However, their MHD counterparts are not widespread, the reason being the higher complexity of the MHD turbulent cascade compared to the purely hydrodynamic cascade. Several subgrid-MHD models have been discussed in the literature including Smagorinskytype models (Smagorinsky 1963; Yoshizawa 1987) which can be used in finite difference/volume codes and Chollet \& Lesieurtype models (Chollet \& Lesieur 1981; Baerenzung et al. 2008) targeted to spectral methods. However, most of these models are still under development and their applicability to $P m \ll 1$ flows is yet to be proven.

In this work we have chosen to use well-known hydrodynamical subgrid models to treat the kinetic turbulent cascade only, leaving the induction equation with standard Ohmic resistivity. This approach is valid provided that the subgrid model is introduced at a scale much smaller than the resistive scale, so that the energy cascade is mostly hydrodynamical. Moreover, it has the advantage of using well-tested subgrid models, which are reasonably simple to implement numerically. This type of method has already been used to study Taylor-Green flows (Ponty et al. 2004) and dynamo action (Ponty et al. 2005) in the limit $P m \rightarrow 0$. We therefore reproduce this approach using very similar tools in the MRI turbulence context.

\subsubsection{Chollet-Lesieur model in incompressible simulations}

Since our incompressible simulations are done with a spectral code, we have used the spectral subgrid model of Chollet \& Lesieur (1981, hereafter CL) to perform our incompressible LES. This model replaces the standard viscosity $v$ by the following expression in Fourier space,

$v_{\mathrm{CL}}(k)=\left(\frac{E\left(k_{\mathrm{c}}\right)}{k_{\mathrm{c}}}\right)^{1 / 2}\left(0.267+9.21 \exp \left[-3.03 k_{\mathrm{c}} / k\right]\right)$

where $k_{\mathrm{c}}$ is the cutoff scale and $E\left(k_{\mathrm{c}}\right)$ is the kinetic energy at the cutoff scale ${ }^{5}$. This expression encloses long-range non-linear interactions via a constant viscosity at $k \ll k_{\mathrm{c}}$ and a cusp due to local energy transfers close to the cutoff scale $k_{\mathrm{c}}$. Interestingly, $k_{\mathrm{c}}$ is the only free parameter of this model. The amount of viscosity itself is automatically adjusted according to the amount of energy at the cutoff scale. It should be emphasized that this expression is only valid for 3D homogeneous and isotropic Kolmogorov turbulence. In principle, it is therefore not applicable to turbulent shear flows found in shearing box models since such flows are anisotropic (see Sect. 3.1.1). As a first attempt to quantify this anisotropy, we proceed as follows. We define a decomposition in spherical harmonics ${ }^{6}$ such that

$E_{K}(\boldsymbol{k})=\frac{1}{2}|\tilde{v}(\boldsymbol{k})|^{2}=\sum_{j m} c_{j}^{m}(|\boldsymbol{k}|) Y_{j m}(\hat{k})$

\footnotetext{
5 Several forms for the CL viscosity may be found in the literature since this expression is a fit to numerical EDQNM (eddy-damped quasi-normal Markovian) calculations. Our expression comes from the asymptotic viscosity of Chollet \& Lesieur (1981) and a fit close to $k_{\mathrm{c}}$ of Sagaut (2006).

6 This use of spherical harmonics to estimate the anisotropy of turbulence is a standard procedure for the study of sheared flows (e.g. Biferale \& Vergassola 2001).
}

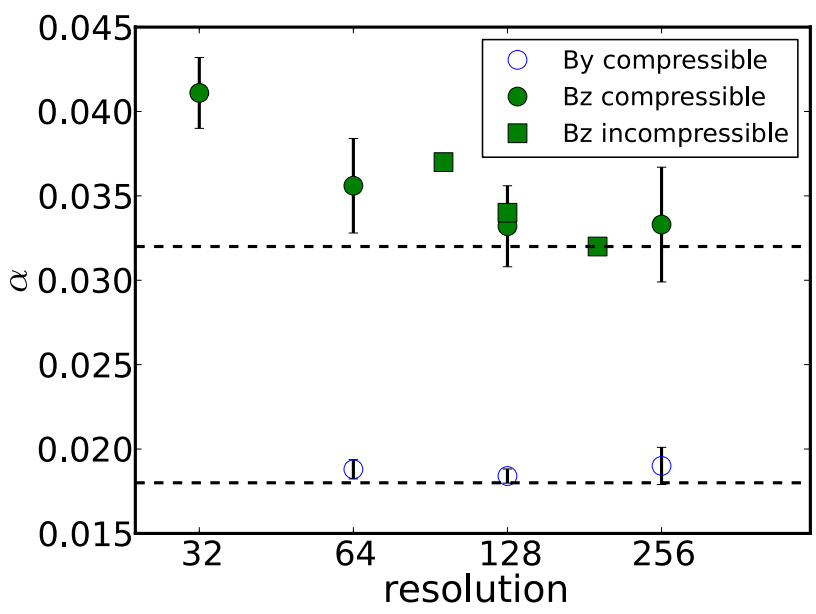

Fig. 6. Mean value of the angular momentum transport (measured by means of $\alpha$ ) for the mean toroidal field LES simulation (implicit LES blue circles), the mean vertical field LES simulation performed with RAMSES (implicit LES, green filled circles), and with SNOOPY (Chollet-Lesieur LES, green filled squares). The resolution is given in number of cells per scale height. The dashed lines represent the asymptotic limits obtained with DNS.

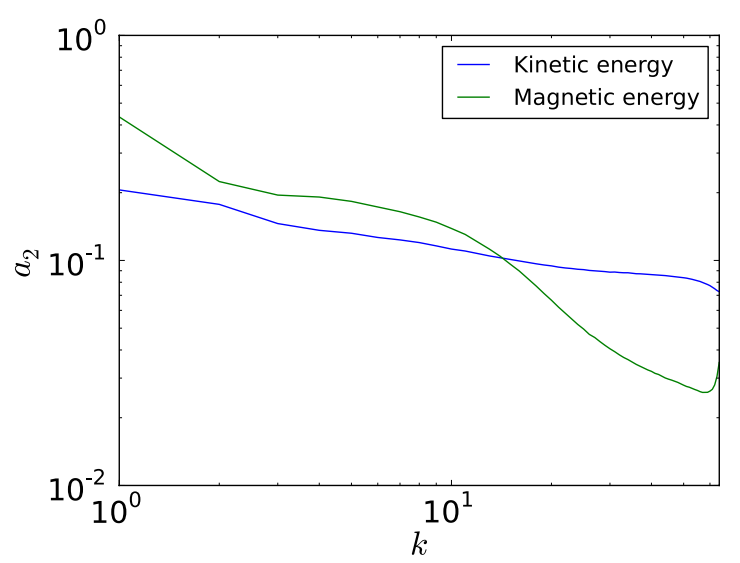

Fig. 7. Estimate of the anisotropy at each scale. For each wavenumber, the energy is decomposed into spherical harmonics; the anisotropy is estimated from the sum of the second-order coefficients normalized by the isotropic coefficient.

and similarly for the magnetic energy. We focus on the $l=2$ coefficients of the decomposition and define:

$a_{2}=\sum_{m=-2}^{m=2}\left|c_{2}^{m}\right|^{2} /\left|c_{0}\right|^{2}$

here $a_{2}$ takes significant values when there are strong variations of the energy over the shell or, in other words, when the flow displays anisotropy at that scale. As shown in Fig. 7, it decreases with $k$, but is still high at small scales for the kinetic energy. Nevertheless, we will see that the Chollet-Lesieur model gives satisfactory results.

We have performed several simulations using the CL subgrid model, varying $k_{\mathrm{c}}$ and the resolution (runs Z-CL-XXX-X). Models labelled $a$ have $k_{\mathrm{c}} / k_{\max }=0.75$ and models labelled $b$ have $k_{\mathrm{c}} / k_{\max }=1$, where $k_{\max }$ is the maximum accessible wavenumber of the simulation (Table 3). As plotted in Fig. 6 (green squares), all of our models recover the statistical results of direct numerical simulations (DNS) with a reduced resolution. These encouraging results are confirmed by turbulent spectra 

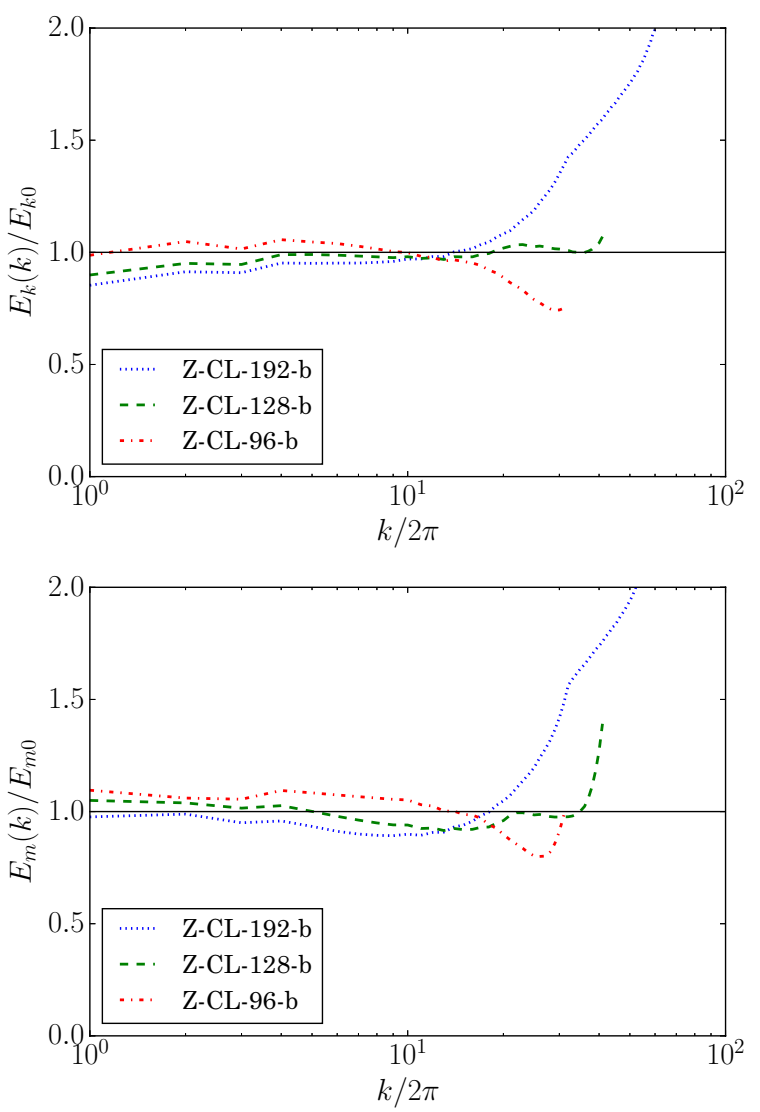

Fig. 8. Ratio of power spectra of kinetic energy (top) and magnetic energy (bottom) obtained with a Chollet-Lesieur subgrid model with $k_{\mathrm{c}} / k_{\max }=1$ and full DNS calculation at $R e=40000$.

compared to DNS simulation (Fig. 8). We find that both kinetic and magnetic spectra agree to less than $10 \%$ down to $k \sim 20$ with the highest resolution DNS Z-I-Re40000. We also find that simulations with $k / k_{\mathrm{c}}=0.75$ exhibit the same convergence properties, at least for the resolution we studied. Therefore, the cutoff scale does not seem to have much impact on these results, provided it is below the resistive dissipation scale. These results demonstrate that CL models can be used efficiently to study low $P m$ flows with a gain in resolution of at least a factor of 2 associated with a gain in computation time of at least a factor of 20.

\subsubsection{Implicit LES in compressible simulations}

In the small Pm limit, the simplest possible subgrid scale model when using finite volume codes is certainly the so-called "Implicit LES" (ILES). The idea is to capture Ohmic resistivity explicitly in the simulation (since it occurs at large scale) but let numerical dissipation handle kinetic energy dissipation at small scales. This method has already been used in some previous works (Fleming et al. 2000; Inutsuka \& Sano 2005; Okuzumi \& Hirose 2011; Flock et al. 2012, 2015), but its range of validity has never been systematically and quantitatively investigated. In this section, we compare the results of such simulations performed with RAMSES at various resolutions with the results presented in Sect. 3.1.

The azimuthal field case: the magnetic Reynolds number is here fixed to $R m=2600$ as it was for DNS. We performed a series of simulations with resolutions ranging from 64 to 256 cells
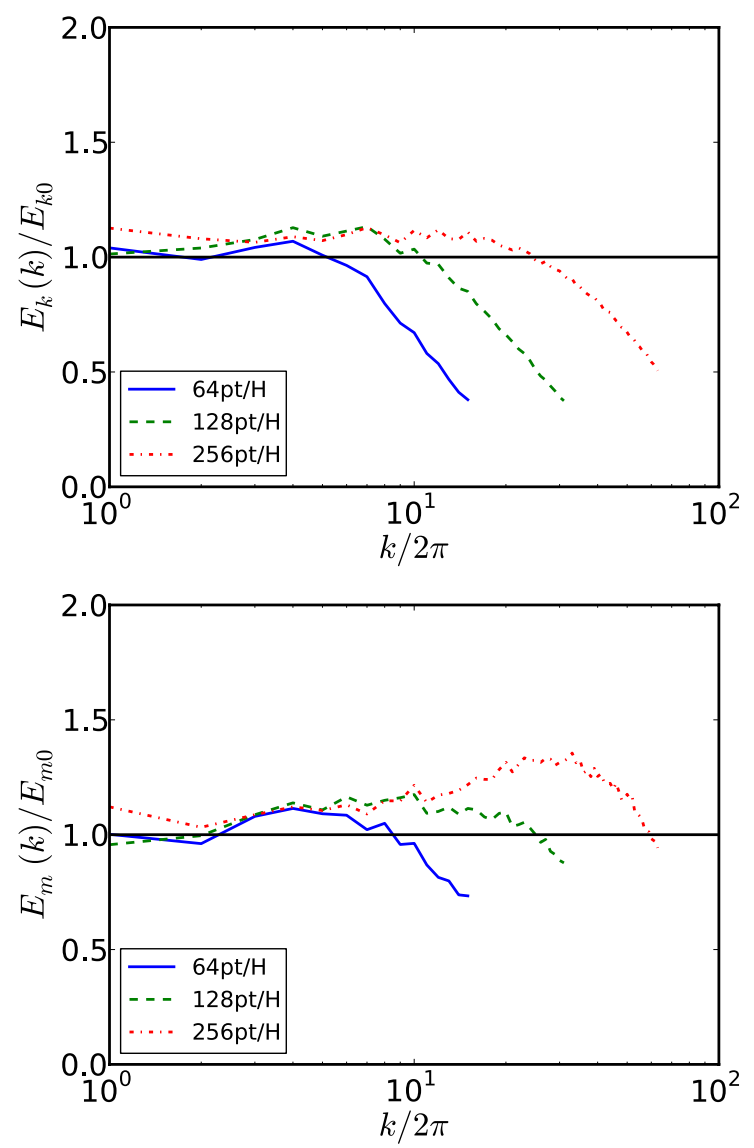

Fig. 9. Ratio between the power spectra obtained with RAMSES in the ILES (green, red, blue, and dotted curves and model Y-C-Re85000 in the azimuthal magnetic field cases. Top panel is for kinetic energy power spectrum and bottom panel for magnetic energy power spectrum. In both panels, the black horizontal line marks the location of the unity ratio.

per scale height. For all cases, we obtain $\alpha=1.8 \times 10^{-2}$, in very good agreement with our best resolved simulations at $P m=0.03$ (see Fig. 6). We then compared the kinetic and magnetic energy power spectra in these simulations with the spectra obtained in the DNS with the lowest Prandlt number (Y-C-Re85000). In the upper panel (resp. lower panel) of Fig. 9, we plot the ratio of the kinetic (resp. magnetic) power spectra between the ILES and the DNS. The runs give an acceptable agreement with the DNS at all available scales (except at small scales in the kinetic energy, which is a result of the different hydrodynamical dissipation): at all resolutions, the deviations to the DNS run for $k^{\prime}<10$ for both the kinetic and the magnetic energy spectra, are at most of the order of $15 \%$.

The vertical field case: the resolution in this case was varied from 32 cells per scale height to 256 cells per scale height. As can be seen in Fig. 6, $\alpha$ decreases as resolution increases, most likely as a result of the decrease of the effective numerical viscosity, and converges to the value determined by the DNS simulations. Quite surprisingly, however, the convergence as resolution is increased toward the asymptotic value of $\alpha$ at low Pm is slower than the azimuthal field case, even though $R m$ is much larger in that case. Indeed, with 64 cells per scale height, the difference between the two $\alpha$ values is still approximately $20 \%$, while it has reached convergence in the case of a mean $B_{y}$. This is probably due to the stress dependence with $\mathrm{Pm}$ being steeper 

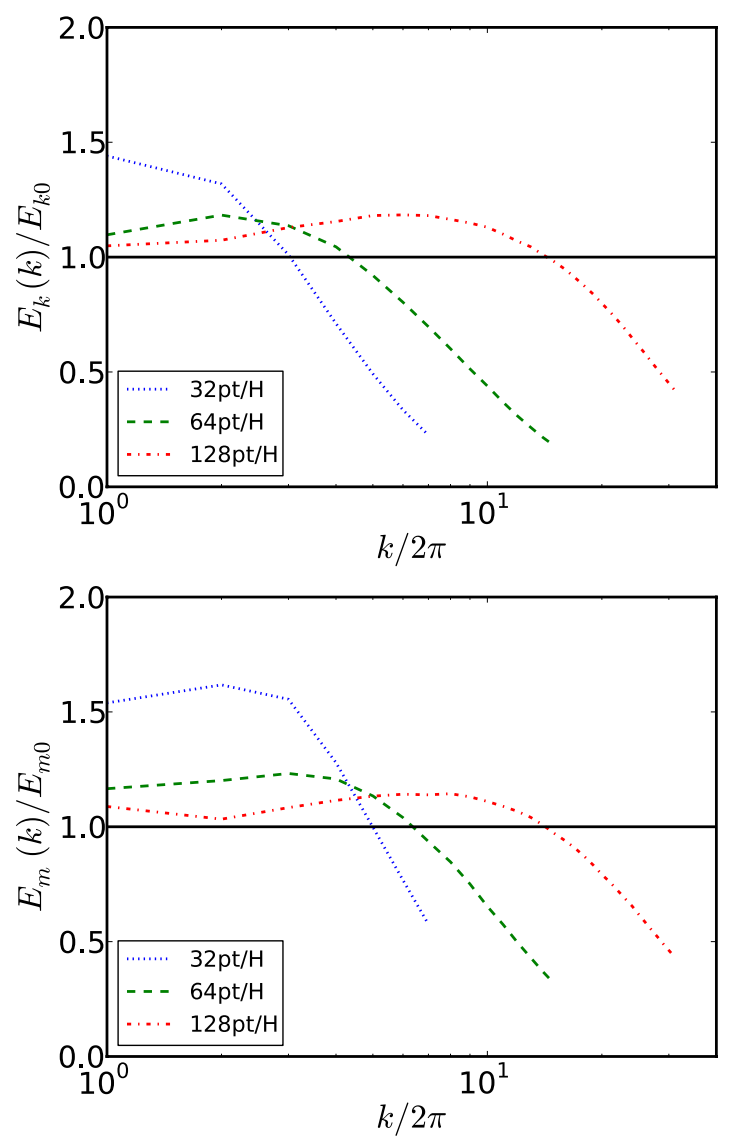

Fig. 10. Same as Fig. 9, but for the vertical field case.

in the presence of a mean vertical field. In any case, 128 cells per scale height are needed to reach the asymptotic $\alpha$ value to less than $10 \%$. We note that this is still a gain of a factor of 16 in computing time. The power spectra displayed in Fig. 10 confirm that result: there is a difference of about $50 \%$ between the power spectra of the ILES and the DNS at large scale for a resolution of 32 cells per scale height. Even the largest resolution reveals differences of up to $20 \%$ in both the kinetic and magnetic power spectra, even if the largest scales are converged to better than $10 \%$, as is expected from the good agreement between the transport coefficients in both simulations (Fig. 6).

\section{Conclusions}

We briefly summarize the main results and ideas of the paper and discuss some of their limitations.

Our main goal was to investigate the properties of the turbulent state in the small magnetic Prandtl number limit by mean of local DNS simulations. We showed that in the presence of a mean magnetic field threading the domain, angular momentum transport converges to a finite value in the small $\mathrm{Pm}$ limit. This result is valid both with a vertical and with an azimuthal mean magnetic field with the asymptotic values at small $\mathrm{Pm}$ being, respectively, $\alpha=1.8 \times 10^{-2}$ with $R m=2600$ and $\beta_{y} \sim 10^{2}$ and $\alpha=3.2 \times 10^{-2}$ with $R m=400$ and $\beta_{z}=10^{3}$. The obtained values with our set of parameters are consistent with the estimations computed from the lifetime and accretion rate of protoplanetary discs ( $\alpha$ is a few $10^{-2}$ ). Obviously, a word of care is in order here: the magnetization of accretion discs, such as protoplanetary discs, is only loosely constrained and the value of the $\beta$ parameter is unknown. The value of $\alpha$ is known to strongly depend on the field strength, with $\alpha$ proportional to $\beta^{-1 / 2}$ (Hawley et al. 1995) or $\beta^{-1}$ (Bodo et al. 2011) in the vertical field case. Similarly, we can expect angular momentum transport to increase with the magnetic Reynolds number (Longaretti \& Lesur 2010). The asymptotic values of the angular momentum rate we obtained are thus only valid for the $\beta$ and $R m$ parameters we considered, and with a scaling that remains to be determined in the small $P m$ limit.

In the case of the compressible simulations with a mean vertical field, we obtained a surprising ratio of Maxwell to Reynolds stress of about 1 . Notwithstanding any possible dependance with $\beta$ and $R m$, we noticed that this ratio is related to the compressibility of the fluid as a more usual value of about 3 is obtained in the incompressible runs. It is also related to the bursty behaviour of these two stresses: whereas a usual value is obtained in the growing phase of the bursts, the Reynolds stress dominates during its decreasing parts, resulting in a mean $R$ value of about 1 . This result is specific to the compressible simulations, but it implies that neither the Reynolds stress nor the Maxwell stress converges to the same value in compressible and incompressible simulations at low Pm. In fact, both stresses differ by about $50 \%$, which we show can be attributed to their different behaviour during the bursts. It is possible that the convergence of $\alpha$ at low Pm to the same value with this two types of flow is fortuitous. In order to solve that issue, higher $\mathrm{Rm}$ simulations are needed but are currently very costly.

Next, we investigated the interest of using large eddy simulations both in spectral and real spaces for such simulations. The simplest approach is to consider the implicit LES method. As expected, in all cases the smallest scales are not correctly handled, but it is possible to reproduce the large-scale energy spectra by using a resolution that is high enough. To obtain an error smaller than $20 \%$ on $\alpha$, the needed resolution corresponds to a fourth of the resolution needed when explicit viscosity is included. This corresponds to a decrease in CPU time of a factor of 256. To limit the computational cost of a simulation of a turbulent flow, explicit subgrid scale (SGS) models are also usually considered. The anisotropy of the rotating sheared flow and the turbulent cascade, which differs from the Kolmogorov cascade, may indicate that simple SGS approaches are not well suited for MRI turbulence simulations. We tested the CholletLesieur method which is used in spectral space and is applied directly on the spectrum components. This method is local in frequency space, the effective viscosity being a function of the wave number, and there is a limited inclusion of the backscatter. We found that good results are also obtained with the CholletLesieur approach notwithstanding the anisotropy of the flow at small scales.

Despite such positive results, a word of care is in order here. Although we showed that these methods are efficient at decreasing the computational cost of MRI turbulence simulations, the needed resolution is still significant. For a magnetic Reynolds number $R m=400$ with a vertical mean magnetic field, the required resolution is $64 \mathrm{pts} / H$. This $R m$ is low compared to the values that are often chosen in the literature for which an even higher resolution is then necessary. To reach a turbulent flow dominated by Ohmic dissipation, a resolution of at least $256 \mathrm{pts} / H$ will be needed for $R m$ values of a few thousands. Moreover, we considered only two diagnostics, namely the angular momentum transport rate and the power spectra, to reach this conclusion and other diagnostics, such as helicity or correlation functions, were not considered. Such statistical quantities may well be incorrectly described in our LES for the resolutions 
we used. More generally, with such reduced resolutions, any small scale process is not correctly handled, and for instance the study of collision of grains in protoplanetary discs or magnetic reconnection cannot be studied in such simulations.

Overall we still conclude that LES can be used to limit the computational time of future simulations. Future works should account for density stratification, which will be particularly relevant in the context of global simulations. Large-scale phenomena such as the ones identified in the "butterflies diagrams" can strongly modify the flow properties and are likely to affect the LES approach. Dedicated simulations should be performed to quantify the interest of such methods in this case.

Acknowledgements. H.M., S.F., and M.J. acknowledge funding from the European Research Council under the European Union's Seventh Framework Programme (FP7/2007-2013)/ERC Grant agreement No. 258729. G.L. acknowledges support by the European Community via contract PCIG09-GA2011-294110. The simulations presented in this paper were granted access to the HPC resources of CCRT under the allocation x2012042231 made by GENCI (Grand Équipement National de Calcul Intensif).

\section{References}

Baerenzung, J., Politano, H., Ponty, Y., \& Pouquet, A. 2008, Phys. Rev. E, 77, 046303

Bai, X.-N. 2015, ApJ, 798, 84

Bai, X.-N., \& Stone, J. M. 2011, ApJ, 736, 144

Bai, X.-N., \& Stone, J. M. 2013, ApJ, 769, 76

Bai, X.-N., \& Stone, J. M. 2014, ApJ, 796, 31

Balbus, S. A. 2003, ARA\&A, 41, 555

Balbus, S. A., \& Hawley, J. F. 1991, ApJ, 376, 214

Balbus, S. A., \& Hawley, J. F. 1998, Rev. Mod. Phys., 70, 1

Balbus, S. A., \& Henri, P. 2008, ApJ, 674, 408

Beresnyak, A. 2014, ApJ, 784, L20

Biferale, L., \& Vergassola, M. 2001, Phys. Fluids, 13, 2139

Bodo, G., Mignone, A., Cattaneo, F., Rossi, P., \& Ferrari, A. 2008, A\&A, 487, 1

Bodo, G., Cattaneo, F., Ferrari, A., Mignone, A., \& Rossi, P. 2011, ApJ, 739, 82

Brandenburg, A., Nordlund, A., Stein, R. F., \& Torkelsson, U. 1995, ApJ, 446, 741

Chollet, J.-P., \& Lesieur, M. 1981, J. Atmosph. Science, 38, 2747

Evans, C. R., \& Hawley, J. F. 1988, ApJ, 332, 659

Fleming, T. P., Stone, J. M., \& Hawley, J. F. 2000, ApJ, 530, 464

Flock, M., Henning, T., \& Klahr, H. 2012, ApJ, 761, 95

Flock, M., Ruge, J. P., Dzyurkevich, N., et al. 2015, A\&A, 574, A68
Frank, J., King, A., \& Raine, D. J. 2002, Accretion Power in Astrophysics: Third Edition (Cambridge University Press)

Frisch, U., Kurien, S., Pandit, R., et al. 2008, Phys. Rev. Lett., 101, 144501

Fromang, S. 2010, A\&A, 514, L5

Fromang, S. 2013, EAS Pub. Ser., 62, 95

Fromang, S., Hennebelle, P., \& Teyssier, R. 2006, A\&A, 457, 371

Fromang, S., Papaloizou, J., Lesur, G., \& Heinemann, T. 2007, A\&A, 476, 1123

Glatzmaiers, G. A., \& Roberts, P. H. 1995, Nature, 377, 203

Guan, X., Gammie, C. F., Simon, J. B., \& Johnson, B. M. 2009, ApJ, 694, 1010

Hawley, J. F., \& Balbus, S. A. 1992, ApJ, 400, 595

Hawley, J. F., Gammie, C. F., \& Balbus, S. A. 1995, ApJ, 440, 742

Hawley, J. F., Gammie, C. F., \& Balbus, S. A. 1996, ApJ, 464, 690

Herault, J., Rincon, F., Cossu, C., et al. 2011, Phys. Rev. E, 84, 036321

Inutsuka, S.-i., \& Sano, T. 2005, ApJ, 628, L155

Johansen, A., Youdin, A., \& Klahr, H. 2009, ApJ, 697, 1269

King, A. R., Pringle, J. E., \& Livio, M. 2007, MNRAS, 376, 1740

Kunz, M. W., \& Lesur, G. 2013, MNRAS, 434, 2295

Latter, H. N., Lesaffre, P., \& Balbus, S. A. 2009, MNRAS, 394, 715

Lesur, G., \& Longaretti, P.-Y. 2007, MNRAS, 378, 1471

Lesur, G., \& Longaretti, P.-Y. 2011, A\&A, 528, A17

Lesur, G., Kunz, M. W., \& Fromang, S. 2014, A\&A, 566, A56

Longaretti, P.-Y., \& Lesur, G. 2010, A\&A, 516, A51

Mason, J., Cattaneo, F., \& Boldyrev, S. 2008, Phys. Rev. E, 77, 036403

Masset, F. 2000, A\&AS, 141, 165

Miyoshi, T., \& Kusano, K. 2005, J. Comput. Phys., 208, 315

Murphy, G. C., \& Pessah, M. E. 2015, ApJ, 802, 139

Okuzumi, S., \& Hirose, S. 2011, ApJ, 742, 65

Ponty, Y., Politano, H., \& Pinton, J.-F. 2004, Phys. Rev. Lett., 92, 144503

Ponty, Y., Mininni, P. D., Montgomery, D. C., et al. 2005, Phys. Rev. Lett., 94, 164502

Riols, A., Rincon, F., Cossu, C., et al. 2013, J. Fluid Mech., 731, 1

Riols, A., Rincon, F., Cossu, C., et al. 2015, A\&A, 575, A14

Sagaut, P. 2006, Large Eddy Simulation for Incompressible Flows (Springer)

Schekochihin, A. A., Cowley, S. C., Taylor, S. F., Maron, J. L., \& McWilliams, J. C. 2004, ApJ, 612, 276

Schekochihin, A. A., Iskakov, A. B., Cowley, S. C., et al. 2007, New J. Phys., 9, 300

Shakura, N. I., \& Sunyaev, R. A. 1973, A\&A, 24, 337

Simon, J. B., \& Hawley, J. F. 2009, ApJ, 707, 833

Simon, J. B., Hawley, J. F., \& Beckwith, K. 2011, ApJ, 730, 94

Simon, J. B., Bai, X.-N., Armitage, P. J., Stone, J. M., \& Beckwith, K. 2013, ApJ, 775, 73

Smagorinsky, J. 1963, Mon. Weather Rev., 91, 99

Stone, J. M., \& Gardiner, T. A. 2010, ApJS, 189, 142

Stone, J. M., Hawley, J. F., Gammie, C. F., \& Balbus, S. A. 1996, ApJ, 463, 656

Teyssier, R. 2002, A\&A, 385, 337

Yoshizawa, A. 1987, Phys. Fluids, 30, 1089 